\title{
Estratégias de enfrentamento em crianças em situação pré-cirúrgica: relação com idade, sexo, experiência com cirurgia e estresse
}

\author{
Coping strategies among children in a pre-surgical \\ situation: Relationship with age, gender, \\ experience with surgery and stress
}

\author{
Luciana Esgalha CARNIER ${ }^{1}$ \\ Flávia Helena Pereira PADOVANI ${ }^{2}$ \\ Gimol Benzaquen PEROSA² \\ Olga Maria Piazentin Rolim RODRIGUES ${ }^{3}$
}

\begin{abstract}
Resumo
O estudo objetivou verificar as estratégias de enfrentamento de crianças, com idade entre 7 e 12 anos, em situação pré-cirúrgica, e sua relação com variáveis sociodemográficas, presença de estresse na criança, no acompanhante e experiência prévia com cirurgia. Para tanto, 58 crianças internadas para realização de cirurgias eletivas responderam ao instrumento de avaliação das estratégias de enfrentamento da hospitalização e a um questionário de avaliação do estresse. O acompanhante respondeu a um levantamento sociodemográfico e ao inventário de sintomas de estresse de Lipp. As estratégias mais utilizadas foram distração e solução do problema e as menos utilizadas, afastamento social e oposição. Meninas e filhos de pais com estresse usaram significativamente mais estratégias de regulação da emoção. Observou-se maior uso de reestruturação cognitiva em crianças mais velhas e com experiência prévia em cirurgia. Os resultados sinalizam a necessidade de considerar as variáveis estudadas quando da elaboração de programas de preparação cirúrgica.
\end{abstract}

Palavras-chave: Coping; Criança hospitalizada; Estresse.

\begin{abstract}
This study's aim was to verify coping strategies among children aged between 7 and 12 years old who will be experiencing surgery and the potential relationship of coping strategies with sociodemographic variables, stress in children and in companions, and the child's prior experience with surgery. A total of 58 children hospitalized and waiting for elective

$\boldsymbol{\nabla} \boldsymbol{\nabla} \boldsymbol{\nabla} \boldsymbol{v}$

${ }^{1}$ Universidade Estadual Paulista Júlio de Mesquita Filho, Faculdade de Medicina, Hospital das Clínicas. Botucatu, SP, Brasil.

2 Universidade Estadual Paulista Júlio de Mesquita Filho, Faculdade de Medicina de Botucatu, Departamento de Neurologia, Psicologia e Psiquiatria. Caixa-Postal 540, Distrito de Rubião Jr., s/n., 18618-970, Botucatu, SP, Brasil. Correspondência para/Correspondence to: F.H.P. PADOVANI.E-mail: <fpadovani@fmb.unesp.br>.

3 Universidade Estadual Júlio de Mesquita Filho, Faculdade de Ciências, Departamento de Psicologia, Bauru, SP, Brasil.
\end{abstract}


surgeries responded to the Assessment Instrument of Coping to Hospitalization and an instrument to assess stress. The companion received a sociodemographic questionnaire and the Lipp Stress Symptom Inventory. Problem-solving strategies and distraction were the most frequent strategies used, while social withdrawal and opposition were the least frequent. Girls and children of stressful parents used emotion regulation strategies with significantly more frequency. Likewise, greater cognitive restructuring was observed in older children and those with prior experience with surgeries. The results show the need to consider the studied variables when developing pre-operative preparation programs.

Keywords: Coping; Child hospitalized; Stress.

A internação hospitalar de crianças é considerada um fator estressor para o desenvolvimento na medida em que há uma alta probabilidade delas serem submetidas a procedimentos invasivos e traumáticos. Dentre os procedimentos invasivos, as experiências cirúrgicas são consideradas fatores de impacto por estarem atreladas ao imprevisível e ao incontrolável, circundadas por fantasias, medos e reações negativas, o que pode provocar elevados níveis de estresse (Carvalho, Silva, Santos, \& Camargo, 2006; Moro \& Módolo, 2004).

Especificamente no período pré-cirúrgico, apesar das reações de medo e ansiedade serem consideradas normais, quando exacerbadas, podem interferir direta ou indiretamente no curso da patologia e da própria cirurgia (Sebastiani \& Maia, 2005). O período que circunda a cirurgia, principalmente aquele que antecede à anestesia, envolve o medo da criança de se separar dos pais, as incertezas quanto aos procedimentos cirúrgicos e seus resultados, provocando ansiedade e temores que podem ser verbalizados pela criança ou que podem levar à alteração de seu comportamento.

Estudos brasileiros focaram os sentimentos e medos de crianças em situação pré-cirúrgica. Em geral, os resultados encontrados confirmam que a cirurgia desperta sentimentos negativos (medo, culpa, tristeza, desconfiança) e intenção de fuga (Crepaldi \& Hackbarth, 2002), decorrentes de temores da anestesia, do ato cirúrgico e da possibilidade de, posteriormente, não poder desempenhar determinadas atividades (Broering \& Crepaldi, 2011).

Para fazer face ao estresse da internação, a criança recorre a esforços adaptativos denominados estratégias de enfrentamento ou coping, determinados tanto pela sua maturação biológica quanto psicológica. A partir de uma visão desenvolvimen- cognitivo e social interferem na capacidade da criança para compreender e significar a doença e em sua capacidade de lançar mão de estratégias de enfrentamento, determinando sua reação adaptativa (Compas, Connor-Smith, Saltzman, Thomsen, \& Wadsworth, 2001).

Segundo Folkman e Lazarus (1985), o enfrentamento pode ser definido como um conjunto de esforços - comportamentais e cognitivos -, utilizado pelo indivíduo para lidar com situações de estresse que sobrecarregam seus recursos pessoais. Os autores identificaram duas funções do coping, uma em que se tentaria controlar ou alterar a situação estressora (coping baseado no problema), e outra que regularia a resposta emocional associada ao estresse ou ao resultado de eventos estressantes (coping baseado na emoção).

Compas et al. (2001), identificaram três dimensões funcionais nas estratégias de enfrentamento. A primeira envolve esforços ativos para alcançar algum controle pessoal sobre os aspectos estressantes do ambiente e sobre sua emoção; a segunda inclui esforços cognitivos para se adaptar à situação como reestruturação, aceitação ou distração, pensamentos e atividades positivas; e, finalmente, a terceira contém respostas de evitação ou de desligamento do fator estressor ou de sua emoção.

Zimmer-Gembeck e Skinner (2011), por sua vez, a partir de análises teóricas e de vários estudos empíricos, chegaram a 12 famílias de coping, em que cada família representa um conjunto de formas funcionalmente homogêneas de estratégias de enfrentamento. Todas as estratégias de determinada família servem para as mesmas funções adaptativas, mas a forma como uma particular função é expressa está intimamente associada às capacidades desenvolvimentais do indivíduo. 
As estratégias de enfrentamento das crianças são mais específicas que do adulto, de acordo com o desenvolvimento de suas habilidades cognitivas, sociais e de regulação da emoção. Pesquisas com crianças mostram que tanto os sentimentos como o uso de diferentes estratégias de enfrentamento estão associados a variáveis como idade e sexo, este último por haver formas de socialização diferentes para meninos e meninas (Allegretti, 2006). Com relação à idade, Wills (1986) verificou que a variabilidade e diversidade dos tipos de enfrentamento aumentavam com a idade.

Para Barros (2003) e Costa Junior (2005), o enfrentamento em que a criança tende a controlar a emoção para reduzir a tensão é mais frequente em crianças mais velhas, bem como o uso de estratégias mais complexas de enfrentamento. As habilidades para enfrentamento focalizado no problema parecem ser adquiridas mais cedo, desenvolvendo-se até aproximadamente 8 a 10 anos de idade. Crianças pequenas, presas a um pensamento concreto, se beneficiam de enfrentamentos comportamentais e busca por apoio emocional, ao passo que crianças mais velhas e jovens, com capacidade para controlar emoções e usar recursos imaginários, podem utilizar o relaxamento ou transformar o significado subjetivo da situação estressante. Barros (2003) conclui que as competências cognitivas envolvidas no enfrentamento emocional tendem a ser mais sofisticadas e só emergem no fim da idade escolar, quando a criança passa a utilizar regras mais abstratas e generalizáveis para controlar a ação.

Apesar da importância do fator idade para prever quais situações a criança considera estressantes e por qual tipo de estratégia vai optar, ele não é um fator suficiente para explicar toda a variabilidade de desempenho das crianças frente ao estresse (Barros, 2003). Outros fatores como temperamento, a possibilidade de controle do estressor, a experiência prévia com dor, cirurgias e procedimentos invasivos, modelos de enfrentamento familiar e o modo como os pais reagem à situação têm um peso determinante na escolha do enfrentamento a que a criança recorre, assim como na sua eficácia (Costa Junior, 2005; Kain, Mayes, Weisman, \& Hofstadter, 2000).
Moro e Módolo (2004) relatam que no momento pré-cirúrgico, filhos de pais ansiosos mostravam-se mais irritados que crianças cujos pais eram mais tranquilos e não apresentavam sinais de ansiedade. Kain et al. (2000) avaliaram o temperamento infantil, as habilidades cognitivas, o comportamento adaptativo e o estilo de enfrentamento de 60 crianças, com idades variando entre 3 e 10 anos, uma semana antes da realização de cirurgia eletiva, assim como ansiedade e o estilo de enfrentamento dos pais e a ansiedade da criança no momento pré-cirúrgico.Os resultados indicaram que a ansiedade dos pais, as habilidades sociais adaptativas e o temperamento da criança (sociabilidade), foram preditores independentes de elevada ansiedade.

Considerando a escassa literatura sobre possíveis variáveis associadas às estratégias de enfrentamento no período pré-cirúrgico, esta pesquisa teve por objetivo verificar as estratégias de enfrentamento de crianças hospitalizadas em situação pré-cirúrgica e sua relação com variáveis sociodemográficas, como idade e sexo, presença de estresse na criança, nos acompanhantes e experiência prévia com cirurgia.

\section{Método}

\section{Participantes}

A amostra foi composta por 58 crianças e um de seus acompanhantes, que: estavam internadas para realização de cirurgias eletivas/agendadas; tinham idade entre 7 anos e 12 anos e 11 meses; e conhecimento da internação para realizar cirurgia. Foram excluídas crianças: internadas para biópsia; portadores de síndromes que comprometiam a capacidade cognitiva e que estavam sob efeito de sedativos ou referiam dor muito acentuada.

A pesquisa foi realizada nas enfermarias do Hospital das Clínicas da Faculdade de Medicina de Botucatu (HC-FMB), onde as crianças estavam internadas. Elas eram admitidas na véspera da cirurgia, no período da tarde e os médicos responsáveis pela 
cirurgia e anestesia visitavam a criança no leito para realização de exames, dar informações e esclarecer dúvidas.

\section{Instrumentos}

a) Questionário de levantamento de características sociodemográficas e das condições atuais das crianças quanto à hospitalização. Solicitava-se ao acompanhante, informações sobre a criança (data de nascimento, sexo e experiência anterior da criança com hospitalizações e cirurgias) e sobre ele próprio (idade, sexo, grau de parentesco);

b) Escala de Stress Infantil (ESI), elaborado por Lipp e Lucarelli (2011) com o objetivo de verificar em crianças a presença de estresse, a fase de estresse em que se encontram (alerta, resistência, quase-exaustão e exaustão) e a sintomatologia prevalente. É composto por 35 afirmações em que a criança avalia cada afirmação, a partir de uma escala Likert de quatro pontos. Quanto às qualidades psicométricas, a escala total apresentou um índice de consistência interna (Alpha de Cronbach) de 0,89;

c) Inventário de Sintomas de Stress para Adultos (ISSL), elaborado por Lipp (2002), tem como finalidade avaliar a presença de estresse em jovens e adultos, a fase de estresse em que se encontram (alerta, resistência, quase exaustão e exaustão) e a sintomatologia prevalente. Contêm 53 afirmações, frente às quais o participante deve assinalar os sintomas experimentados em três momentos diversos, seguindo uma ordem hierárquica de intensidade e gravidade de sintomas. As qualidades psicométricas da escala mostraram-se satisfatórias, com índice de consistência interna (Alpha de Cronbach) de 0,91.

d) Instrumento de Avaliação das Estratégias de Enfrentamento da Hospitalização (AEH). Instrumento elaborado para identificar as estratégias a que recorriam crianças hospitalizadas para tratamento de câncer. Em sua última versão o AEHcomp é composto por um roteiro de entrevista e um caderno (um para meninos e outro para meninas), com 20 cenas coloridas que retratam possíveis comporta322 brincar, assistir TV, cantar, ouvir música, rezar, estudar, conversar, ler gibi, tomar remédio, buscar informações, chorar, sentir raiva, sentir-se triste, desanimar, sentir medo, pensar em fugir, chantagem, esconder, sentir culpa, pensar em milagre (Motta \& Enumo, 2010). Pede-se à criança que aponte se emitiu o comportamento, em que ocasiões e com que frequência. Para facilitar a resposta, são oferecidos à criança retângulos de tamanhos diferentes (em escala crescente). A seguir, pede-se que a criança justifique porque utiliza, ou não, cada comportamento.

\section{Procedimentos}

As crianças hospitalizadas para cirurgias eletivas e seus responsáveis foram abordados, pelo menos quatro horas após a internação, tempo considerado suficiente para que a criança já tivesse vivenciado o ambiente hospitalar. Se aceitassem participar, solicitava-se que o acompanhante assinasse o Termo de Consentimento Livre e Esclarecido. Crianças com 11 anos ou mais, também assinavam o termo.

O acompanhante respondia ao questionário de levantamento de características sociodemográficas e ao ISSL, sem a presença da criança e em local com garantia de privacidade. A seguir, aplicavam-se os instrumentos com a criança, sem a presença do acompanhante e, se possível, não à beira do leito. Quando permitido, as respostas eram gravadas. O estudo teve aprovação do Comitê de Ética da Universidade Estadual Paulista Júlio de Mesquita Filho, Campus Bauru (Processo no 1596/46/01/08) e do HC-FMB (Of. 350/08-CEP).

Inicialmente, as escalas para avaliação de estresse foram corrigidas e pontuadas conforme as respectivas normas. Para a análise das respostas ao $\mathrm{AEH}$, em uma primeira etapa foram computadas as frequências de cada comportamento que a criança informou utilizar. A seguir, foram selecionados os cinco comportamentos mais utilizados e os cinco menos utilizados e, as justificativas das respostas a cada comportamento foram categorizadas segundo as estratégias de enfrentamento adaptadas para o contexto da hospitalização por Motta (2007), a par- 
tir das formulações de Skinner, Edge, Altman e Sherwood (2003), conforme descrito na Tabela 1. Como muitas crianças tiveram dificuldade em justificar o porquê de alguns comportamentos, criou-se uma categoria denominada "nenhuma justificativa".

Apesar das cenas do AEH estarem direta ou indiretamente relacionadas à determinada estratégia de enfrentamento, era a justificativa da criança que levava à categorização da resposta. Por exemplo: se a criança, ao ser indagada porque assistia TV, justificasse "porque é gostoso", ou, "porque o tempo passa mais rápido", se computava como estratégia de distração. No entanto, se a resposta fosse que assistia TV para se "sentir mais calma", era computada como regulação da emoção; ou ainda, se justificasse que fazia isto "para não pensar na cirurgia", era considerada uma estratégia de esquiva.

Dependendo do número de justificativas dadas, cada comportamento emitido, podia ser categorizado em mais de uma estratégia. A seguir, calculou-se a frequência média das estratégias utilizadas pelas crianças, primeiramente no grupo como um todo, e, posteriormente, nos grupos de crianças divididos segundo idade, gênero, presença de estresse, experiência prévia com cirurgia e presença de estresse no acompanhante. Finalmente, passou-se à análise estatística inferencial. Para a comparação das médias dos grupos foram utilizados os testes não paramétricos para dois grupos independentes de Mann Whitney e, para mais de dois grupos, o teste de Kruskal-Wallis para grupos

Tabela 1

Descrição das categorias de estratégias de enfrentamento

\begin{tabular}{|c|c|}
\hline Estratégia & Descrição \\
\hline Solução de Problemas & $\begin{array}{l}\text { Ações dirigidas para a resolução do problema, comportamentos de adesão ao tratamento (ingestão dos } \\
\text { remédios prescritos, submissão a exames e procedimentos médicos); indicação de foco no tratamento e na } \\
\text { cura da doença. }\end{array}$ \\
\hline Busca por Suporte & $\begin{array}{l}\text { Ação em direção ao alvo de suporte (familiar, profissional da equipe hospitalar, voluntário, outra criança, } \\
\text { pesquisador e Deus), assim como a aceitação do suporte social disponível. }\end{array}$ \\
\hline Esquiva & $\begin{array}{l}\text { Tentativas de não envolvimento com a situação estressante, mantendo-se distante dela. Inclui "fuga cognitiva", } \\
\text { como não pensar no problema, negação e ações diretas de evitação. }\end{array}$ \\
\hline Distração & $\begin{array}{l}\text { Engajamento em atividades alternativas prazerosas e possíveis no ambiente hospitalar, como forma de tentar } \\
\text { lidar com a situação estressante. }\end{array}$ \\
\hline Reestruturação Cognitiva & $\begin{array}{l}\text { Tentativas ativas para mudar sua percepção sobre a situação estressante, no sentido de vê-la de uma maneira } \\
\text { mais positiva; esforços para minimizar o próprio sofrimento ou as consequências negativas da hospitalização/ } \\
\text { cirurgia. }\end{array}$ \\
\hline Ruminação & $\begin{array}{l}\text { Foco passivo e repetitivo nos aspectos negativos da situação, com ênfase nos danos e perdas da situação } \\
\text { estressante, podendo também ser presente catastrofização, autoculpa e medo. }\end{array}$ \\
\hline Desamparo & Passividade, resignação, confusão, interferência ou exaustão cognitiva, desânimo e pessimismo. \\
\hline Afastamento Social & $\begin{array}{l}\text { Ações dirigidas a manter-se distante das outras pessoas ou impedi-las de saber sobre a situação estressante ou } \\
\text { seus efeitos emocionais. }\end{array}$ \\
\hline Regulação da Emoção & $\begin{array}{l}\text { Esforços para influenciar o sofrimento emocional e expressar as emoções construtivamente no momento e } \\
\text { lugar apropriados, fazendo com que se sinta melhor, utilizando, por exemplo, autoencorajamento, controle } \\
\text { emocional, e expressão emocional. }\end{array}$ \\
\hline Busca por Informação & $\begin{array}{l}\text { Tentativas de aprender mais sobre a situação estressante, por meio de perguntas diretas ou da observação dos } \\
\text { acontecimentos. }\end{array}$ \\
\hline Negociação & $\begin{array}{l}\text { Tentativas ativas de fazer um acordo entre suas necessidades, o fato de ser submetida a uma cirurgia e as } \\
\text { restrições impostas pelo contexto da hospitalização. }\end{array}$ \\
\hline Oposição & Comportamentos de objeção, agressão, reação de raiva, descarga e atribuição de culpa às outras pessoas. \\
\hline Delegação & Comportamentos de dependência, busca por ajuda mal adaptativa, reclamações, queixas e autopiedade. \\
\hline
\end{tabular}


independentes. Os resultados foram considerados significativos a um nível de 5\%. Para os cálculos e testes, empregou-se o Software Statistica da StatSoft (Siegel, 1981).

\section{Resultados}

Das crianças avaliadas, 22 (37,9\%) tinham idade entre 7 e 8 anos e 11 meses, 12 (20,7\%) de 9 a 10 anos e 11 meses, e $24(41,4 \%)$ de 11 a 12 anos e 11 meses. A maioria era meninos (53,4\%), metade apresentou estresse e 58,6\% não tinham experiência prévia com cirurgia.

Os acompanhantes, em sua grande maioria, eram os cuidadores primários $(97,0 \%)$, do sexo feminino (90,0\%), sendo que $77,6 \%$ pontuaram para estresse, a maioria na fase de resistência $(73,3 \%)$ e a sintomatologia prevalente foi psicológica (93,3\%).
Na Figura 1, pode-se observar que os comportamentos que as crianças disseram emitir mais frequentemente foram tomar remédio, assistir TV, conversar, rezar e brincar e os cinco menos frequentes foram esconder-se, pensar em fugir, sentir culpa, fazer chantagem e desanimar.

A Figura 2 mostra que as estratégias de enfrentamento com maiores médias foram Distração (DIS) e Solução de Problemas (SPR). Em contrapartida, as estratégias com médias mais baixas foram Afastamento Social (ASO) e Oposição (OPO). Entretanto, a média de "nenhuma justificativa" foi a mais alta $(M=2,28)$.

A média de estratégias de Regulação da Emoção (REM) $(p=0,02)$ e Ruminação (RUM) $(p=0,002)$ das meninas foi, significativamente, mais alta que dos meninos. As crianças mais novas, com idade entre 7 e 8 anos, apresentaram maior dificuldade para justificar seus comportamentos que

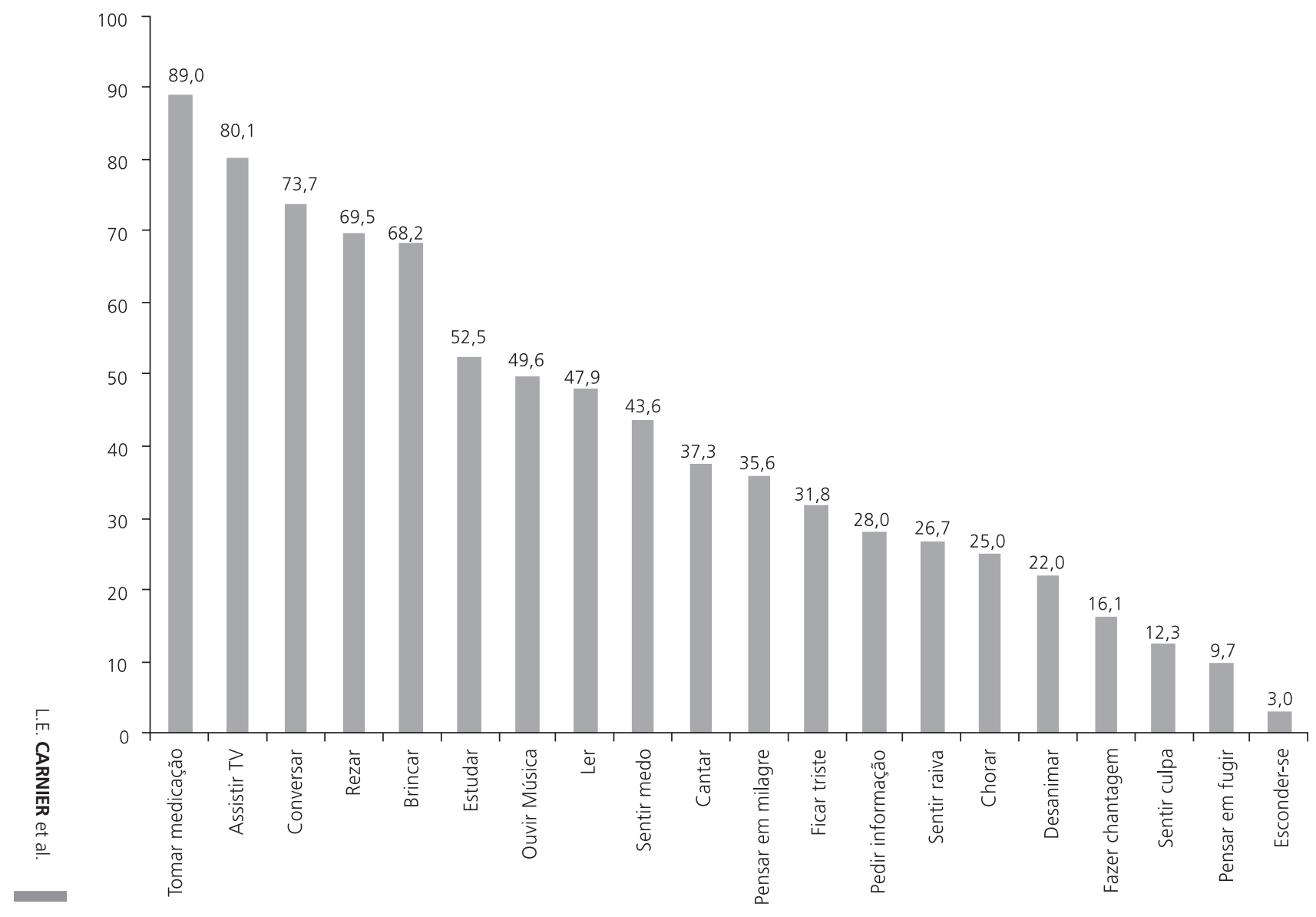

324 Figura 1. Porcentagem de utilização dos comportamentos de enfrentamento da hospitalização de crianças em situação pré-cirúrgica ( $\mathrm{N}=58$ ). 
as mais velhas $(p \leq 0,001)$, além de relatarem menos estratégias de Reestruturação Cognitiva (RCO) ( $p=0,001)$. O grupo de crianças com experiência anterior com procedimentos cirúrgicos teve, de forma significativa, média mais alta da estratégia de

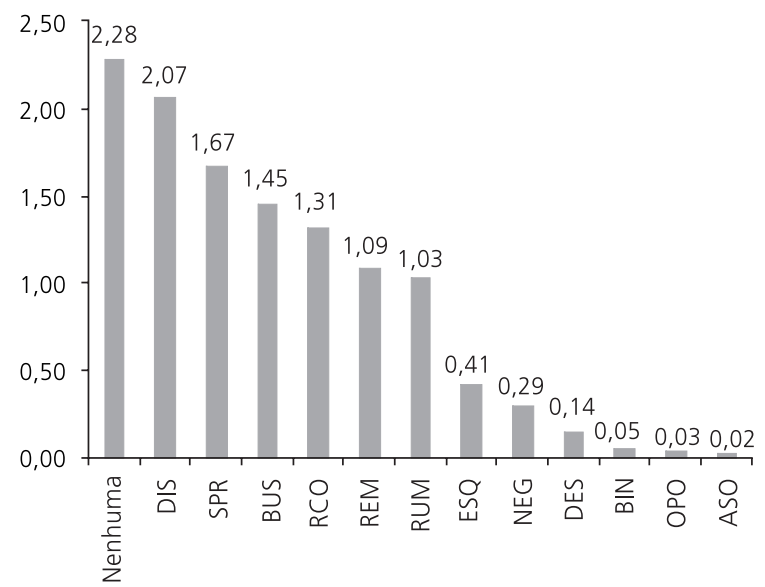

Figura 2. Média das estratégias de enfrentamento utilizadas pelas crianças em situação pré-cirúrgica ( $N=58)$.

Nota: DIS: Distração; SPR: Solução de Problemas; BUS: Busca por Suporte; RCO: Reestruturação Cognitiva; REM: Regulação da Emoção; RUM: Ruminação; ESQ: Esquiva; NEG: Negação; DES: Desamparo; BIN: Busca por Informação; OPO: Oposição; ASO: Afastamento Social. reestruturação cognitiva $(p=0,002)$, que crianças sem experiência cirúrgica (Tabela 2).

Não houve diferenças estatisticamente significativas nas estratégias de enfrentamento das crianças com e sem estresse. Por outro lado, as crianças cujos acompanhantes pontuaram para estresse apresentaram médias significativamente maiores de estratégias de REM $(p=0,004)$ e RUM $(p=0,015)$ que crianças de cuidadores sem estresse (Tabela 3 ).

\section{Discussão}

Confirmando a literatura (Amitay et al., 2006; Broering \& Crepaldi, 2011; Moro \& Módolo, 2004; Sebastiani \& Maia, 2005), a internação para realizar uma cirurgia eletiva caracterizou-se como uma situação estressante para metade das crianças e para $77 \%$ dos pais.

A prevalência de estresse no acompanhante foi maior do que a encontrada com cuidadores de crianças portadoras de quadros crônicos, como câncer (Del Bianco Faria \& Cardoso, 2010; Patiño-Fernandez et al., 2008), possivelmente porque os pais das crianças crônicas estão, há algum tempo,

Tabela 2

Comparações entre as médias das estratégias de enfrentamento de crianças pré-cirúrgicas divididas quanto a sexo, idade e experiência prévia com cirurgia $(N=58)$

\begin{tabular}{|c|c|c|c|c|c|c|c|c|c|c|}
\hline \multirow[b]{2}{*}{$\begin{array}{l}\text { Estratégias de } \\
\text { enfrentamento }\end{array}$} & \multicolumn{3}{|c|}{$\operatorname{Sexo}^{a}$} & \multicolumn{4}{|c|}{ Idade $^{\mathbf{b}}$} & \multicolumn{3}{|c|}{ Experiência prévia com cirurgia ${ }^{a}$} \\
\hline & $\begin{array}{l}\text { Meninas } \\
(\mathrm{N}=27)\end{array}$ & $\begin{array}{l}\text { Meninos } \\
(\mathrm{N}=31)\end{array}$ & Valor $p$ & $\begin{array}{c}7-8 \\
\text { anos } \\
(\mathrm{N}=22)\end{array}$ & $\begin{array}{c}9-10 \\
\text { anos } \\
(\mathrm{N}=12)\end{array}$ & $\begin{array}{c}11-12 \\
\text { anos } \\
(\mathrm{N}=24)\end{array}$ & Valor $p$ & $\begin{array}{c}\text { Sem } \\
\text { experiência } \\
(N=34)\end{array}$ & $\begin{array}{c}\text { Com } \\
\text { experiência } \\
(\mathrm{N}=24)\end{array}$ & Valor $p$ \\
\hline DIS & 2,1 & 2,1 & 0,977 & 2,0 & 2,0 & 2,2 & 0,888 & 2,1 & 2,1 & 0,943 \\
\hline REM & 1,4 & 0,8 & $0,020^{*}$ & 1,1 & 1,3 & 1,0 & 0,559 & 1,0 & 1,2 & 0,427 \\
\hline BUS & 1,3 & 1,5 & 0,463 & 1,3 & 1,6 & 1,5 & 0,640 & 1,4 & 1,5 & 0,591 \\
\hline RUM & 1,4 & 0,7 & $0,002^{*}$ & 0,8 & 1,3 & 1,1 & 0,312 & 1,1 & 1,0 & 0,819 \\
\hline DES & 0,1 & 0,1 & 0,844 & 0,1 & 0,1 & 0,2 & 0,474 & 0,2 & 0,0 & 0,095 \\
\hline ESQ & 0,5 & 0,3 & 0,237 & 0,4 & 0,6 & 0,3 & 0,532 & 0,5 & 0,3 & 0,096 \\
\hline ASO & 0,0 & 0,0 & & 0,0 & 0,0 & 0,0 & & 0,0 & 0,0 & \\
\hline OPO & 0,0 & 0,0 & & 0,1 & 0,0 & 0,0 & & 0,0 & 0,0 & \\
\hline $\mathrm{RCO}$ & 1,5 & 1,2 & 0,254 & 0,7 & 1,6 & 1,8 & $0,002^{*}$ & 0,9 & 1,8 & $0,002^{*}$ \\
\hline SPR & 1,6 & 1,7 & 0,797 & 1,4 & 1,7 & 1,9 & 0,346 & 1,8 & 1,5 & 0,350 \\
\hline BIN & 0,0 & 0,1 & 0,105 & 0,1 & 0,1 & 0,0 & & 0,1 & 0,0 & 0,145 \\
\hline NEG & 0,3 & 0,3 & 0,592 & 0,3 & 0,3 & 0,3 & 0,951 & 0,4 & 0,2 & 0,309 \\
\hline Nenhuma estratégia & 2,0 & 2,5 & 0,093 & 3,3 & 1,8 & 1,5 & $\leq 0,001^{*}$ & 2,2 & 2,3 & 0,782 \\
\hline
\end{tabular}

Nota: ${ }^{*} p \leq 0,05$. ${ }^{a}$ Comparações por meio do teste de Mann-Whitney; ${ }^{\mathbf{b}}$ Comparações por meio do teste de Kruskall-Wallis.

DIS: Distração; SPR: Solução de Problemas; BUS: Busca por Suporte; RCO: Reestruturação Cognitiva; REM: Regulação da Emoção; RUM: Ruminação; ESQ:

Esquiva; NEG: Negação; DES: Desamparo; BIN: Busca por Informação; OPO: Oposição; ASO: Afastamento Social. 
Tabela 3

Comparações entre as médias das estratégias de enfrentamento de crianças pré-cirúrgicas divididas quanto ao estresse da criança e do cuidador $(N=58)$

\begin{tabular}{|c|c|c|c|c|c|c|}
\hline \multirow{2}{*}{$\begin{array}{l}\text { Estratégias de } \\
\text { enfrentamento }\end{array}$} & \multicolumn{3}{|c|}{ Estresse criança } & \multicolumn{3}{|c|}{ Estresse acompanhante } \\
\hline & $\begin{array}{l}\text { Sem estresse } \\
\qquad(N=29)\end{array}$ & $\begin{array}{l}\text { Com estresse } \\
\qquad(\mathrm{N}=29)\end{array}$ & Valor $p$ & $\begin{array}{l}\text { Sem estresse } \\
\qquad(N=13)\end{array}$ & $\begin{array}{l}\text { Com estresse } \\
\qquad(\mathrm{N}=45)\end{array}$ & Valor $p$ \\
\hline DIS & 2,0 & 2,1 & 0,987 & 2,3 & 2,0 & 0,446 \\
\hline REM & 1,1 & 1,0 & 0,538 & 0,4 & 1,3 & $0,004^{*}$ \\
\hline BUS & 1,5 & 1,3 & 0,486 & 1,3 & 1,5 & 0,605 \\
\hline RUM & 0,7 & 1,3 & 0,089 & 0,5 & 1,2 & $0,015^{*}$ \\
\hline DES & 0,1 & 0,2 & 0,221 & 0,1 & 0,2 & 0,498 \\
\hline ESQ & 0,3 & 0,5 & 0,161 & 0,4 & 0,4 & 0,850 \\
\hline ASO & 0,0 & 0,0 & & 0,0 & 0,0 & \\
\hline OPO & 0,0 & 0,0 & & 0,0 & 0,0 & \\
\hline $\mathrm{RCO}$ & 1,2 & 1,4 & 0,491 & 1,2 & 1,3 & 0,760 \\
\hline SPR & 2,0 & 1,4 & 0,082 & 1,7 & 1,7 & 0,945 \\
\hline BIN & 0,1 & 0,1 & 1,000 & 0,0 & 0,1 & \\
\hline NEG & 0,2 & 0,3 & 0,391 & 0,2 & 0,3 & 0,632 \\
\hline Nenhuma estratégia & 2,4 & 2,2 & 0,558 & 3,2 & 2,0 & $0,007^{*}$ \\
\hline
\end{tabular}

Nota: * $p \leq 0,05$; Comparações por meio do teste de Mann-Whitney.

DIS: Distração; SPR: Solução de Problemas; BUS: Busca por Suporte; RCO: Reestruturação Cognitiva; REM: Regulação da Emoção; RUM: Ruminação; ESQ: Esquiva; NEG: Negação; DES: Desamparo; BIN: Busca por Informação; OPO: Oposição; ASO: Afastamento social.

se adaptando ao diagnóstico e tratamento, enquanto que o momento da cirurgia, agudiza nos pais os medos, a ansiedade e, muitas vezes, os confronta com uma situação em que eles não têm experiência anterior.

Carnier, Rodrigues e Padovani (2012) observaram que a presença significativa de estresse em acompanhantes de crianças hospitalizadas para a realização de cirurgia eletiva estava relacionada a não ter experiência prévia com cirurgia. Diante de tais constatações, Shirley, Thompson, Kenward e Johnston (1998) preconizam que poder acompanhar a criança no tratamento, estar presente durante a indução da anestesia e receber mais informações da equipe no período pré-operatório poderiam contribuir para a redução do estresse paterno.

Quanto à criança, se a hospitalização provoca a manifestação de reações adversas como o estresse, a necessidade de ser submetido a procedimentos invasivos, como as cirurgias, potencializa essas reações (Broering \& Crepaldi, 2011). Mesmo quando se trata de cirurgias eletivas, antes do procedimento, a criança pode experimentar ameaça à sua integridade física, acompanhada do medo da morte e, no pós-cirúrgico, sintomas pós- -traumáticos leves que podem se manter por um mês (Amitay et al., 2006).

Frente à situação estressante, as crianças do presente estudo recorreram a estratégias de enfrentamento para se adaptar, especialmente, distração e solução de problemas. No entanto, devido à dificuldade que as crianças tiveram em justificar suas escolhas, principalmente as mais novas (7 e 8 anos), vários comportamentos não foram categorizados segundo a perspectiva de análise proposta por Motta (2007).

A distração compreendeu atividades passíveis de serem realizadas no ambiente hospitalar, como assistir TV, brincar e ouvir música, consideradas prazerosas, que entretinham e, portanto, aliviavam o estresse. Trata-se de uma estratégia de enfrentamento frequentemente utilizada por crianças hospitalizadas (Moraes \& Enumo, 2008; Motta, 2007; Santos \& Silva, 2002; Wollin et al., 2004).

Na pesquisa de Santos e Silva (2002), a porcentagem de crianças com síndrome nefrótica e doença celíaca, que relataram o desejo de dormir e brincar com outras crianças, foi maior do que das crianças saudáveis. Segundo as autoras, brincar, uma atividade prazerosa, estaria compensando o 
mal-estar e desconforto causados pela doença. Ao comparar as estratégias de enfrentamento de crianças entre 4 e 6 anos, Salmela, Salanterä, Ruotsalainen e Aronen (2010) verificaram que o número de crianças hospitalizadas que apontou o brincar como estratégia de enfrentamento era significativamente maior que as crianças saudáveis de pré-escola. Para Azevedo, Santos, Justino, Miranda e Simpson (2008), a necessidade de brincar, além de ser uma estratégia de distração, é uma atividade conhecida, que potencializa a capacidade da criança se sentir mais segura quando está em um ambiente estranho, com pessoas desconhecidas.

Na preparação pré-cirúrgica utilizam-se livros de histórias, contos infantis, atividades verbais, e exercícios de respiração, como parte da técnica de distração. Por meio desta técnica a criança dirige sua atenção para atividades diferentes daquelas que provocam dor ou mesmo elevam seu nível de ansiedade (Broering \& Crepaldi, 2011). Os próprios sujeitos reconhecem que a distração pode aliviar o estresse pré-cirúrgico. No estudo de Wollin et al. (2004), crianças hospitalizadas para realização de cirurgia eletiva, com idade entre 5 e 12 anos, e seus pais, sugeriram as estratégias de distração, especialmente assistir TV, para diminuir a ansiedade pré-cirúrgica.

Com relação à estratégia solução de problemas, a ação instrumental mais citada pelas crianças foi tomar remédio, justificada como a forma mais rápida para sarar ou atendendo à orientação de um adulto responsável. Possivelmente essa resposta sofreu a influência do momento, já que na fase pré-cirúrgica a criança é submetida a dietas, exames e outros procedimentos preparatórios que implicam na tomada de medicações. Essa resposta também era esperada, pois estudos anteriores já haviam identificado que as crianças hospitalizadas atribuíam propriedades mágicas ao remédio pra obter a cura, ou mesmo justificavam sua ação como de obediência e confiança na autoridade, tanto dos pais quanto dos médicos (Moraes \& Enumo, 2008; Perosa \& Gabarra, 2004; Zimmer-Gembeck \& Skinner, 2011). Reforçando essa constatação, estratégias consideradas socialmente pouco aceitáveis, por prejudicar a colaboração nos procedimentos médicos, como o afastamento social e a oposição, foram as estratégias que as crianças menos referiram utilizar.

No tocante às diferenças entre sexos, as meninas relataram mais estratégias de regulação da emoção e ruminação do que os meninos, confirmando evidências na literatura sobre a utilização de comportamentos e estratégias de enfrentamento diversas, dependendo do sexo, em crianças e adolescentes (Broderick \& Korteland, 2002; Borges, Manso, Tomé, \& Matos, 2008; Hampel \& Petermann, 2005). Allegretti (2006) salienta que essas diferenças no uso das estratégias de enfrentamento podem estar relacionadas a diferentes formas de socialização para meninas e meninos. Estes seriam socializados para serem mais independentes e elas para usarem mais estratégias pró-sociais, como a regulação da emoção. Na adolescência, as diferenças encontradas entre os sexos parecem indicar um perfil mais introvertido, autodirecionado, no caso das meninas e um perfil mais extrovertido, voltado para a busca de apoio externo por parte dos meninos (Câmara \& Carlotto, 2007). Reforçando a associação entre crenças culturais e estratégias de enfrentamento, um estudo com adolescentes verificou que, tanto os meninos quanto as meninas, acreditavam que os meninos não deveriam utilizar a ruminação, uma estratégia mais introspectiva, como forma de enfrentamento (Broderick \& Korteland, 2002).

As diferenças na percepção do evento estressor, tanto da cirurgia como da dor envolvida no procedimento, também podem ter influenciado a utilização de diferentes estratégias de enfrentamento por meninos e meninas. Sundblad, Saartok e Engström (2007) verificaram que a autopercepção de dor e de problemas de saúde diminuía entre os meninos e aumentava entre as meninas, com o aumento da idade. Três anos depois, mais da metade dessas crianças e adolescentes (67\%) respondeu a um novo questionário e confirmou-se um aumento das queixas entre as meninas e diminuição entre os meninos (Sundblad, Jansson, Saartok, Renström, \& Engström, 2008). Em outro estudo, com adolescentes, as meninas referiram mais ansiedade antecipatória no período pré-cirúrgico e mais dor no pe- 
ríodo pós-cirúrgico do que os meninos (Logan \& Rose, 2004).

Com relação à variável estresse do cuidador, neste estudo, também houve diferenças significativas nas estratégias de enfrentamento utilizadas pelas crianças de pais que pontuaram e não pontuaram para estresse. Segundo Doca e Costa Junior (2007), pesquisas em psicologia pediátrica apontam o comportamento dos pais como principal modelo condicionador do comportamento da criança, especialmente quando se espera colaboração dela para a execução de exames e procedimentos terapêuticos. Pais e acompanhantes, com comportamentos caracterizados pela baixa tolerância e concorrentes com o tratamento, podem configurar uma condição de ansiedade que se generaliza para a criança.

A relação entre a ansiedade dos pais e das crianças, no pré-operatório, já foi identificada na literatura (Moro \& Módolo, 2004; Kain, Mayes, Wang, Caramico, \& Hofstadter, 1998; Broering \& Crepaldi, 2011). Especificamente no momento da indução anestésica, Moro e Módolo (2004) relatam que crianças mais ansiosas e menos cooperativas eram as que estavam acompanhadas por pais extremamente ansiosos. Frente a essas evidências conclui-se que os médicos, em especial os anestesistas, deveriam estar atentos não só às características dos pacientes cirúrgicos (socialmente desadaptados, tímidos e inibidos), como também à família, principalmente aos pais ansiosos.

Crianças cujos acompanhantes estavam estressados utilizaram, significativamente, mais estratégias de regulação da emoção e ruminação que crianças de pais sem estresse. A cirurgia da criança, para os pais, é um momento de vulnerabilidade e risco sobre a qual não têm previsibilidade e controle (Crepaldi, Rabuske, \& Gabarra, 2006) e, possivelmente, as crianças passam a compartilhar o desamparo vivenciado pelos pais. Se, de um lado, as crianças compartilharam com os pais as reações de catastrofização e medo que caracterizam a ruminação, de outro, pareceram conseguir controlar o sofrimento emocional, com estratégias de autoencorajamento, controle e expressão positiva das emo328 ções, típicas da regulação da emoção (Motta, 2007).
As crianças com experiência cirúrgica prévia utilizaram mais a estratégia de reestruturação cognitiva em comparação àquelas sem experiência. Provavelmente, ter experienciado situações e sentimentos semelhantes, como dor e medo de se separar dos pais, influencia a forma como a criança lida com a nova situação (Compas et al., 2001; Moro \& Módolo, 2004). As crianças com doenças crônicas, por exemplo, e com história de repetidas internações, são hábeis em identificar e implementar um repertório de estratégias de enfrentamento, como o autoencorajamento, devido à familiaridade e ao conhecimento prévio do contexto hospitalar (Boyd \& Hunsberger, 1998).

Além da experiência prévia com cirurgia, a idade se mostrou um fator importante para o uso da reestruturação cognitiva, reforçando o que a literatura aponta como o curso desenvolvimentista do enfrentamento (Zimmer-Gembeck \& Skinner, 2011). As crianças mais novas, entre 7 e 8 anos, relataram significativamente menos uso de tal estratégia, em comparação às crianças mais velhas, que possuem capacidades cognitivas e emocionais mais sofisticadas e, portanto, recorrem ao uso de regras mais abstratas e generalizáveis para controlar a ação.

Concluindo, os resultados desta pesquisa evidenciaram diferenças significativas das estratégias de enfrentamento utilizadas pelas crianças, dependendo do sexo, idade, experiência anterior com cirurgia e estresse do acompanhante.

Na medida em que crianças mais velhas e com mais experiência recorreram a estratégias mais complexas que as mais novas, como a reestruturação cognitiva, o presente estudo confirma a perspectiva desenvolvimentista das estratégias de enfrentamento, enfatizando o aspecto processual da construção de diferentes formas de controlar o estresse.

No entanto, é preciso ressaltar que se a maioria das crianças conseguiu apontar os comportamentos utilizados para lidar com o estresse pré-cirúrgico, uma alta porcentagem de respostas teve de ser inutilizada, especialmente das crianças mais novas (7 a 8 anos), quando elas não conseguiam justificar o porquê da escolha, impossibilitando 
identificar sua função. Essa constatação cria a necessidade de novos estudos, com o objetivo de rever a possibilidade e a melhor maneira de identificar estratégias de enfrentamento em crianças menores. Apesar dessa limitação, os resultados obtidos permitem considerações para a elaboração de programas de preparação cirúrgica sinalizam a importância de considerar o estágio cognitivo da criança e seu sexo, assim como a necessidade de inserir o acompanhante nas programações, a fim de aumentar as chances de sucesso, reduzindo o nível de estresse frente à cirurgia, da criança e da família.

\section{Referências}

Allegretti, J. (2006). Nivel de stress, fontes estressoras e estratégias de enfrentamento em mulheres (Dissertação de mestrado não-publicada). Pontifícia Universidade Católica de Campinas.

Amitay, G. B., Kosov, I., Reiss, A., Toren, P., Yoran-Hegesh, R., Kotler, M., \& Mozes, T. (2006). Is elective surgery traumatic for children and their parents? Journal of Paediatrics and Child Health, 42(10), 618-624. http:// dx.doi.org/10.1111/j.1440-1754.2006.00938.x

Azevedo, D. M., Santos, J. J. S., Justino, M. A. R., Miranda, F. A. N., \& Simpson, C. A. (2008). O brincar enquanto instrumento terapêutico: opinião dos acompanhantes. Revista Eletrônica de Enfermagem, 10(1), 137-144. Recuperado em novembro 7, 2014, de http://www. fen.ufg.br/fen_revista/v10/n1/v10n1a12.htm

Barros, L. (2003). Psicologia pediátrica: perspectiva desenvolvimentista ( $2^{\mathrm{a}}$ ed). Lisboa: Climepsi.

Borges, A. I., Manso, D. S., Tomé, G., \& Matos, M. G. (2008). Ansiedade e coping em crianças e adolescentes: diferenças relacionadas com a idade e gênero. Análise Psicológica, 4(26), 551-561.

Boyd, J. R., \& Hunsberger, M. (1998). Chronically ill children coping with repeated hospitalizations: Their perceptions and suggested Interventions. Journal of Pediatric Nursing, 13(6), 330-342. http://dx.doi.org/ 10.1016/S0882-5963(98)80021-3

Broderick, P. C., \& Korteland, C. (2002). Coping style and depression in early adolescence: Relationships to gender, gender role, and implicit beliefs. Sex Roles, 46(7/8), 201-213. http://dx.doi.org/10.1023/A:10 19946714220

Broering, C. V., \& Crepaldi, M. A. (2011). Preparação psicológica e o estresse de crianças submetidas a cirurgias. Psicologia em Estudo, 16(1), 15-23. http:// dx.doi.org/10.1590/S1413-73722011000100003

Câmara, S. G., \& Carlotto, M. S. (2007). Coping e gênero em adolescentes. Psicologia em Estudo, 12(1), 87-93.
http://dx.doi.org/10.1590/S1413-73722007000 100011

Carnier, L. E., Rodrigues, O. M. P. R., \& Padovani, F. H. P. (2012). Stress materno e hospitalização infantil précirúrgica. Estudos de Psicologia (Campinas), 29(3), 315-325. http://dx.doi.org/10.1590/S0103-166X20 12000300002

Carvalho, L. S., Silva, C. A., Santos, A. C. O., \& Camargo, C. L. (2006). A criança lidando com estressores précirúrgicos: estudo exploratório. Online Brazilian Journal of Nursing, 5(3). http://dx.doi.org/10.5935/1676-42 85.2006508

Compas, B. E., Connor-Smith, J. K, Saltzman, H., Thomsen, A. H., \& Wadsworth, M. E. (2001). Coping with stress during childhood and adolescence: Problems, progress, and potential in theory and research. Psychological Bulletin, 127(1), 87-127. http:// dx.doi.org/10.1037/0033-2909.127.1.87

Costa Junior, A. L. (2005). Psicologia da saúde e desenvolvimento humano: o estudo do enfrentamento em crianças com câncer e expostas a procedimentos médicos invasivos. In M. A. Dessen \& A. L. Costa Junior (Orgs.), A Ciência do desenvolvimento humano: tendências atuais e perspectivas futuras (pp.171-189). Porto Alegre: Artmed.

Crepaldi, M. A., \& Hackbarth, I. D. (2002). Aspectos psicológicos de crianças hospitalizadas em situação pré-cirúrgica. Temas em Psicologia, 10(2), 99-112.

Crepaldi, M. A., Rabuske, M. M., \& Gabarra, L. M. (2006). Modalidades de atuação do psicólogo em psicologia pediátrica. In M. A. Crepaldi, B. M. Linhares, \& G. B. Perosa (Orgs.), Temas em psicologia pediátrica (pp.13-55). São Paulo: Casa do Psicólogo.

Del Bianco Faria, A. M., \& Cardoso, C. L. (2010). Aspectos psicossociais de acompanhantes cuidadores de crianças com câncer: stress e enfrentamento. Estudos de Psicologia (Campinas), 27(1), 13-20. http://dx.doi. org/10.1590/S0103-166X2010000100002

Doca, F. N. P., \& Costa Junior, A. L. (2007). Preparação psicológica para admissão hospitalar de crianças: uma breve revisão. Paidéia, 17(37), 167-179. http://dx.doi. org/10.1590/S0103-863X2007000200002

Folkman, S., \& Lazarus, R. S. (1985). Analysis of coping in a middle-age community sample. Journal of Health and Social Behavior, 21(3), 219-239. http://dx.doi.org/ 10.1037/0882-7974.2.2.171

Hampel, P., \& Petermann, F. (2005). Age and gender effects on coping in children and adolescents. Journal of Youth and Adolescence, 34(2), 73-83. http://dx. doi.org/10.1007/s10964-005-3207-9

Kain, Z. N., Mayes, L. C., Wang, S., Caramico, L. A., \& Hofstadter, M. B. (1998). Parental presence during induction of anesthesia versus sedative premedication: Which intervention is more effective? Anesthesiology, 89(5), 1147-1156. 
Kain, Z. N., Mayes, L. C., Weisman, S. J., \& Hofstadter, M. B. (2000). Social adaptability, cognitive abilities, and other predictors for children's reactions to surgery. Journal of Clinical Anesthesia, 12(7), 549-554. http:// dx.doi.org/10.1016/S0952-8180(00)00214-2

Lipp, M. E. N. (2002). Inventário de Sintomas de Stress para Adultos (ISSL) ( $2^{a}$ ed. rev.). São Paulo: Casa do Psicólogo.

Lipp, M. E. N., \& Lucarelli, M. D. M. (2011). ESI: Escala de Stress Infantil ( $2^{a}$ ed. rev.). São Paulo: Casa do Psicólogo.

Logan, D. E., \& Rose, J. B. (2004). Gender differences in post-operative pain and patient controlled analgesia use among adolescent surgical patients. Pain, 109(3), 481-487. http://dx.doi.org/10.1016/j.pain.2004.02.0 26

Moraes, E. O., \& Enumo, S. R. F. (2008). Estratégias de enfrentamento da hospitalização em crianças avaliadas por instrumento informatizado. Psico - USF, 13(2), 221-231. http://dx.doi.org/10.1590/S1413-8271200 8000200009

Moro, E. T., \& Módolo, N. S. P. (2004). Ansiedade, a criança e os pais. Revista Brasileira de Anestesiologia, 54(5), 728-738. http://dx.doi.org/10.1590/S0034-7094200 4000500015

Motta, A.B. (2007). Brincando no hospital: uma proposta de intervenção psicológica para crianças hospitalizadas com câncer (Tese de doutorado não-publicada). Universidade Federal do Espírito Santo, Vitória.

Motta, A. B., \& Enumo, S. R. F. (2010). Intervenção psicológica lúdica para o enfrentamento da hospitalização para crianças com câncer. Psicologia: Teoria e Pesquisa, 26(3), 445-454. http://dx.doi.org/10.1590/s0 102-37722010000300007

Patiño-Fernandez, A. M., Pai, A. L. H., Alderfer, M., Hwang, W-T., Reilly, A., \& Kazak, A. E. (2008). Acute stress in parents of children newly diagnosed with cancer. Pediatric Blood \& Cancer, 50(2), 289-292. http:// dx.doi.org/10.1002/pbc.21262

Perosa, G. B., \& Gabarra, L. M. (2004). Explicações de crianças internadas sobre a causa das doenças: implicações para a comunicação profissional de saúde-paciente. Interface, 8(14), 135-147. http://dx.doi.org/ 10.1590/S1414-32832004000100008

Salmela, M., Salanterä, S., Ruotsalainen, T., \& Aronen, E. T. (2010). Coping strategies for hospital-related fears in pre-school-age children. Journal of Paediatrics and Child Health, 46(3), 108-114. http://dx.doi.org/10.1111/ j.1 440-1754.2009.01647.x
Santos, S. V., \& Silva, A. L. (2002). A criança com síndrome nefrótica e com doença celíaca: percepções relativas ao controle da doença e à forma como lida com ela. Análise Psicológica, 20(2), 243-260.

Sebastiani, R. W., \& Maia, E. M. C. (2005). Contribuições da psicologia da saúde-hospitalar na atenção ao paciente cirúrgico. Acta Cirúrgica Brasileira, 20(1), 50-55. http://dx.doi.org/10.1590/S0102-86502005000 700010

Shirley, P. J., Thompson, N., Kenward, M., \& Johnston, G. (1998). Parental anxiety before elective surgery in children. Anaesthesia, 53(10), 956-959. http://dx.doi. org/10.1046/j.1365-2044.1998.00533.x

Siegel, S. (1981). Estatística não paramétrica (para ciências do comportamento). São Paulo: McGraw-Hill.

Skinner, E. A., Edge, K., Altman, J., \& Sherwood, H. (2003). Searching for the structure of coping: A review and critique of category systems for classifying ways of coping. Psychological Bulletin, 129(2), 216-269.

Sundblad, G. M., Saartok, T., \& Engstrom, L-M. T. (2007). Prevalence and co-occurrence of self-rated pain and perceived health in school-children: Age and gender differences. European Journal of Pain, 11(2), 171-180. http://dx.doi.org/10.1016/j.ejpain.2006.02.006

Sundblad, G. B., Jansson, A., Saartok, T., Renström, P., \& Engström, L. M. (2008). Self-rated pain and perceived health in relation to stress and physical activity among school-students: A 3-year follow-up. Pain, 136(3), 239-249. http://dx.doi.org/10.1016/j.pain.2007.0 6.032

Wills, T. (1986). Stress and coping in early adolescence: Relationships to substance abuse in urban school samples. Health Psychology, 5(6), 503-529. http://dx. doi.org/10.1037/0278-6133.5.6.503

Wollin, S. R., Plummer, J. L., Owen, H., Hawkins, R. M. F., Materazzo, F., \& Morrison, V. (2004). Anxiety in children having elective surgery. Journal of Pediatric Nursing, 19(2), 128-132. http://dx.doi.org/10.1016/ S0882-5963(03)00146-5

Zimmer-Gembeck, M. J., \& Skinner, E. A. (2011). The development of coping across childhood and adolescence: An integrative review and critique of research. International Journal of Behavioral Development, 35(1), 1-17. http://dx.doi.org/10.1177/0 165025410384923

Recebido: dezembro 11, 2014

Aprovado: janeiro 12, 2015 